\title{
Breast milk fatty acid profile in relation to infant growth and body composition: results from the INFAT study
}

\author{
Daniela Much', Stefanie Brunner', Christiane Vollhardt', Daniela Schmid' ${ }^{1}$ Eva-Maria Sedlmeier ${ }^{2,3}$, Monika Brüderl', \\ Ellen Heimberg ${ }^{1,5}$, Nana Bartke ${ }^{6}$, Günther Boehm ${ }^{6,7}$, Bernhard L. Bader ${ }^{2,3}$, Ulrike Amann-Gassner ${ }^{1}$ and Hans Hauner ${ }^{1,2}$
}

BACKGROUND: There is some evidence that the $n-6 / n-3$ long-chain polyunsaturated fatty acids (LCPUFAs) ratio in early nutrition, and thus in breast milk, could influence infant body composition.

METHODS: In an open-label randomized controlled trial (RCT), 208 healthy pregnant women were allocated to a dietary intervention (supplementation with 1,200 mg n-3 LCPUFAs per day and instructions to reduce arachidonic acid (AA) intake) from the 15th wk of gestation until 4 mo of lactation or to follow their habitual diet. Breast milk LCPUFAs at 6 wk and 4 mo postpartum were related to infant body composition assessed by skinfold thickness (SFT) measurements and ultrasonography during the first year of life.

RESULTS: Dietary intervention significantly reduced breast milk n-6/n-3 LCPUFAs ratio. In the whole sample, early breast milk docosahexaenoic acid (DHA), eicosapentaenoic acid (EPA), and n-3 LCPUFAs at 6 wk postpartum were positively related to the sum of four SFT measurements at age 1. Breast milk AA and n- 6 LCPUFAs at 6 wk postpartum were negatively associated with weight, BMI, and lean body mass (LBM) up to 4 mo postpartum.

CONCLUSION: Breast milk n-3 LCPUFAs appear to stimulate fat mass growth over the first year of life, whereas AA seems to be involved in the regulation of overall growth, especially in the early postpartum period.

$\mathbf{T}$ he amount of n-3 long-chain polyunsaturated fatty acids (LCPUFAs) in human breast milk is subject to maternal nutrition and further modified by maternal adipose tissue stores (1), gestational age and stage of lactation $(2,3)$, and the individual's genetic constitution (variants in the FADS1/2 gene cluster) $(4,5)$. There is an ongoing debate about whether the amount of n-3 LCPUFAs, and particularly the proportions of docosahexaenoic acid (DHA, 22:6n-3), provided to the developing infant during the breastfeeding period may result in functional differences of health outcomes in the offspring.

As recently reviewed, there is insufficient evidence from randomized controlled trials (RCTs) to support or refute the practice of giving n-3 LCPUFA supplementation ("fish oil") to breastfeeding mothers to improve infant growth, children's neurodevelopment, visual acuity, or allergy risk (6).

An upcoming question in the context of fetal programming of later disease susceptibility is whether the balance between the $n-6$ and n-3 LCPUFAs in early nutrition, and thus in breast milk, could play a role in childhood obesity (7). Although prenatal and/or postnatal n-3 LCPUFA supplementation has been shown to reduce obesity in rodents $(8,9)$, although not consistently proven $(10,11)$, evidence in humans is limited and restricted to a few post hoc studies using in large part inadequate methods to assess infant fat mass (12).

The INFAT (The Impact of Nutritional Fatty acids during pregnancy and lactation for early human Adipose Tissue development) study addresses the question as to whether lowering the n-6/n-3 long-chain fatty acid (FA) ratio in the diet of pregnant and breastfeeding women may reduce adipose tissue growth early in life and could, thereby, offer a completely novel approach for the primary prevention of childhood obesity (13). This hypothesis was originally proposed by Ailhaud et al. based on data from various sources suggesting a proadipogenic effect of arachidonic acid (AA) and a counteracting effect of n-3 LCPUFAs (14).

We recently published the clinical results of this prospective randomized RCT examining adipose tissue growth up to the first year of the infant's life. We could not verify the initial hypothesis because we found no intervention effect on fat mass development in the offspring (15).

The purpose of the present analysis was to determine the effect of circulating proportions of n-6 and n-3 LCPUFAs and their ratio in breast milk on measures of infant growth and body composition within the first year of life.

\section{RESULTS}

Breast milk samples were available from $n=152$ women at 6 wk postpartum and $n=120$ women at 4 mo postpartum. The FA profile in red blood cells (RBCs) was measured in a subsample of 56 infants at 4 mo postpartum and 31 infants at 12 mo postpartum. 
Maternal Breast Milk FA Profile Over the Course of Lactation The dietary intervention resulted in significantly higher levels of DHA and eicosapentaenoic acid (EPA), as well as higher proportions of a-linolenic acid, n-3 docosapentaenoic acid, PUFAs, and total n-3 LCPUFAs in breast milk at 6 wk postpartum as compared with those of the control group (Table 1).

By contrast, breast milk contents of dihomo $\gamma$-linolenic acid, adrenic acid, and monounsaturated FAs were significantly reduced in the intervention group as compared with the control group at $6 \mathrm{wk}$ postpartum. Basically, the same FA profile was measured in breast milk taken at 4 mo postpartum as shown in Table 1.
Breast milk proportions of AA did not differ between the groups throughout lactation.

The n-6/n-3 LCPUFA ratio was significantly reduced in the intervention group as compared with the control group throughout the entire lactation period $(P<0.001$, Table 1$)$.

\section{Relationship Between the FA Composition of Breast Milk and Maternal Blood}

Breast milk contents of DHA, EPA, AA, n-3 LCPUFA, and n-6 LCPUFA, as well as the AA/DHA ratio and the n-6/n-3 LCPUFA ratio at $6 \mathrm{wk}$ postpartum were significantly positively correlated with their respective LCPUFAs in maternal plasma

Table 1. Breast milk fatty acid profile

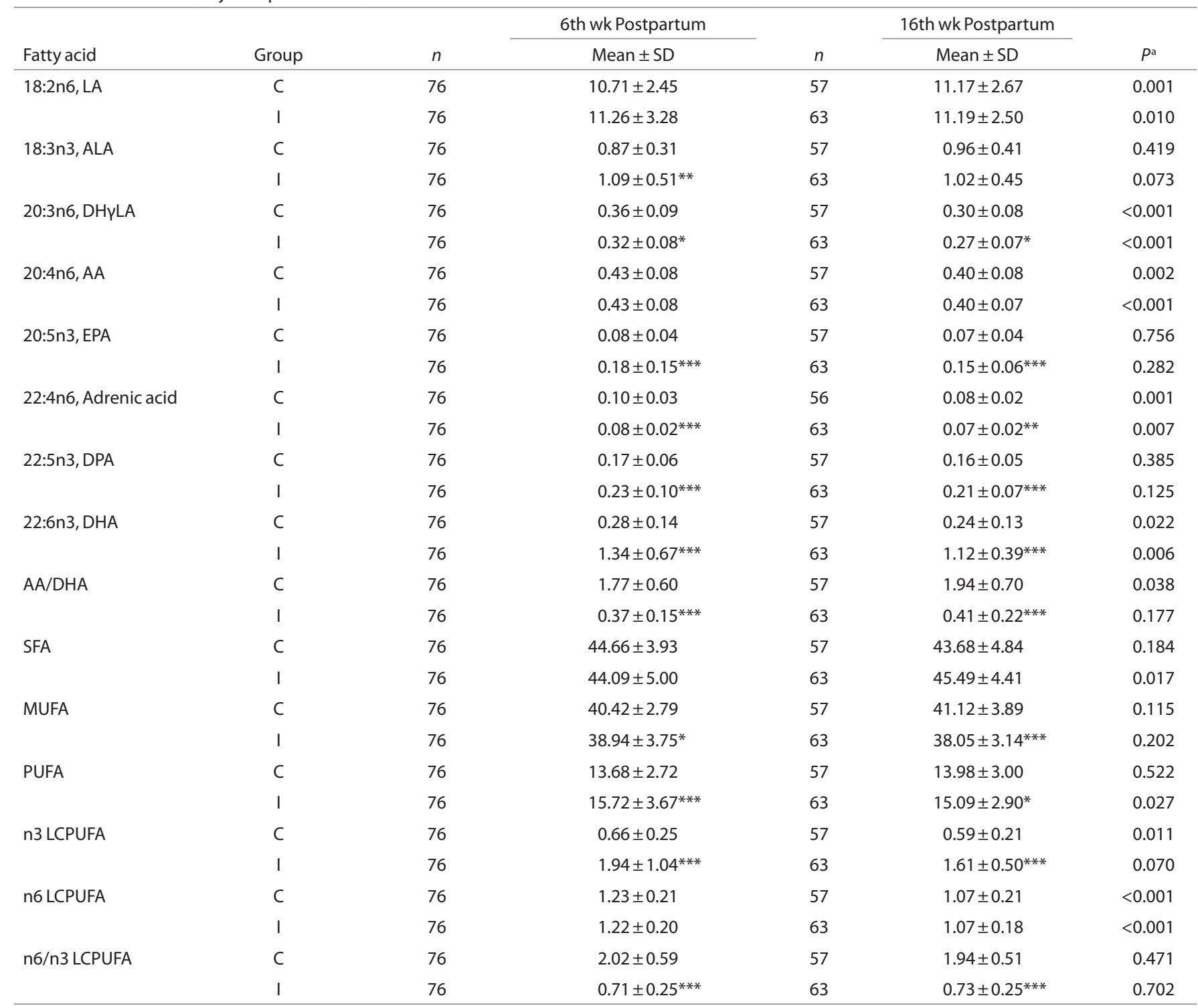

Data are presented as mean \pm SD. Values for fatty acids are expressed as a percentage of the weight of the total fatty acids (\% FA of total FA). Differences in fatty acid values between the groups were compared using the Mann-Whitney U-test. Values marked with asterisks show significant differences between groups at the same time point (Mann-Whitney U-test, $\left.{ }^{*} P<0.05 ;{ }^{*} P<0.01 ; * * P<0.001\right)$ after Bonferroni corrections. SFA: C4:0 - C27:0, MUFA: sum of all cis-FAs with one double bond, PUFA: sum of all cis-FAs with two or more double bonds, n6 LCPUFA: C20:2n6; 20:3n6; 20:4n6; C22:2n6; C22:4n6; C22:5n6, n3 LCPUFA: C20:3n3; C20:4n3; 20:5n3; C21:5n3; C22:3n3; 22:5n3; 22:6n3.

AA, arachidonic acid; ALA, a-linolenic acid; C, control; DHA, docosahexaenoic acid; DHYLA, dihomo Y-linolenic acid; DPA, docosapentaenoic acid; EPA, eicosapentaenoic acid; I, intervention; LA, linolenic acid; LCPUFA, long-chain polyunsaturated fatty acid; MUFA, monounsaturated fatty acid; SFA, saturated fatty acid.

${ }^{a}$ Changes in maternal fatty acid profile over time were determined by using the Wilcoxon test. 
phospholipids at $6 \mathrm{wk}$ and 4 mo postpartum. This relationship also held for RBC FA, albeit to a lesser extent (Supplementary Table S1 online). There was no considerable association between maternal RBC n-6 LCPUFAs and breast milk n-6 LCPUFA level. The maternal breast milk n-6/n-3 LCPUFA ratio was highly correlated with the maternal erythrocyte and plasma phospholipid n-6/n-3 LCPUFA ratio at 6 wk postpartum and 4 mo postpartum (e.g., breast milk approximates maternal plasma phospholipids at 4 mo postpartum: $r=0.941 ; P<0.001$ ).

\section{Infant Blood FA Profile in RBCs}

Infants born to mothers of the intervention group did not significantly differ in their RBC contents of DHA, EPA or AA at
4 and 12 mo postpartum as compared with the control group (Table 2). However, the n-6/n-3 LCPUFA ratio was significantly lower in infants of the intervention group at 4 mo postpartum, whereas the AA/DHA ratio was significantly lower at both time points, 4 and 12 mo postpartum (Table 2).

\section{Relationship Between the FA Composition of Breast Milk and Infant RBCs}

There was no significant correlation between breast milk FA contents of DHA, EPA, or AA at 4 mo postpartum and their respective FAs in infant RBCs at 4 and 12 mo postpartum (Supplementary Table S2 online). There was a significant positive correlation between the n-6/n-3 LCPUFA ratio in

Table 2. Infant RBC fatty acid profile

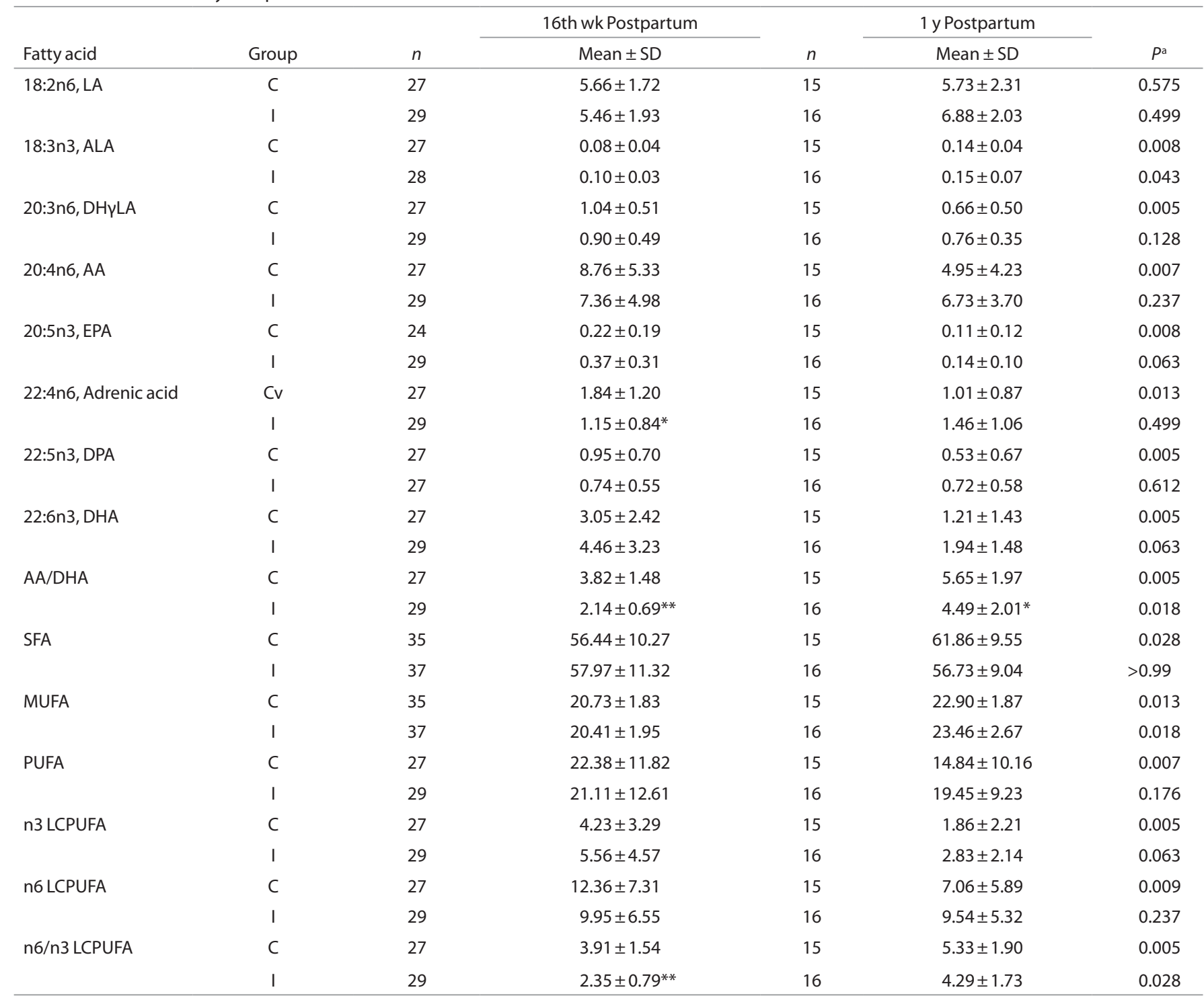

Data are presented as mean \pm SD. Values for fatty acids are expressed as a percentage of the weight of the total fatty acids (\% FA of total FA). Differences in fatty acid values between the groups were compared using the Mann-Whitney U-test.Values marked with asterisks show significant differences between groups at the same time point (Mann-Whitney U-test, ${ }^{*} P<0.05$; $\left.{ }^{*} P<0.001\right)$ after Bonferroni corrections. SFA: C4:0 - C27:0, MUFA: sum of all cis-FA with one double bond, PUFA: sum of all cis-FA with two or more double bonds, $n 6$ LCPUFA: C20:2n6; 20:3n6; 20:4n6; C22:2n6; C22:4n6; C22:5n6, n3 LCPUFA:C20:3n3; C20:4n3; 20:5n3; C21:5n3; C22:3n3; 22:5n3; 22:6n3.

AA, arachidonic acid; ALA, a-linolenic acid; C, control; DHA, docosahexaenoic acid; DHYLA, dihomo -linolenic acid; DPA, docosapentaenoic acid; EPA, eicosapentaenoic acid; I, intervention; LA, linolenic acid; LCPUFA, long-chain polyunsaturated fatty acid; MUFA, monounsaturated fatty acid; RBC, red blood cell; SFA, saturated fatty acid.

aChanges in infant fatty acid profile over time were determined by using the Wilcoxon test. 
breast milk at 4 mo of lactation and the n-6/n-3 LCPUFA ratio in infant erythrocytes at 4 mo postpartum $(n=51 ; r=0.505, P$ $=0.0002)$ and even at 12 mo postpartum $(n=29 ; r=0.387 ; P$ $=0.039$ ). Significant positive correlations were also found for the breast milk AA/DHA ratio at 4 mo postpartum and infant $\mathrm{RBC}$ AA/DHA ratio at $4 \mathrm{mo}(n=51 ; r=0.619 ; P<0.0001)$ as well as at 12 mo postpartum $(n=29 ; r=0.467 ; P=0.012)$.

\section{Early Breast Milk FA Profile (6 wk Postpartum) in Relation to Infant} Growth and Body Composition

There were significant positive associations between DHA, EPA, and total n-3 LCPUFAs and different measures of infant fat mass across all time points: DHA, EPA, and total n-3 LCPUFAs in early breast milk were significantly positively associated with the sum of four skinfold thickness (SFT) measurements at $1 \mathrm{y}$ of age, and for DHA also at 4 mo postpartum (Table 3).

Furthermore, the n-3 FAs were positively associated with some individual skinfolds at the different time points over the first year of life (data not shown): e.g., DHA, EPA, and n-3 LCPUFAs in early breast milk were significantly positively related to biceps SFT in 4 mo olds $(r=0.14, P=0.035$; $r=0.16, P=0.042 ; r=0.15, P=0.037)$, whereas DHA and $\mathrm{n}-3$ LCPUFAs were significantly positively related with triceps SFT in $1-y$ olds $(r=0.24, P=0.034 ; r=0.25, P=0.041)$. Although only a trend, there were positive associations between breast milk EPA and triceps SFT $(r=0.23, P=0.054)$, subscapular SFT $(r=0.16, P=0.090)$, suprailiac SFT $(r=0.13, P=0.092)$, percentage body fat $(r=0.18, P=0.056)$, and fat mass $(\mathrm{g})$ ( $r=0.23, P=0.077)$ at 1 y postpartum. Early breast milk DHA and n-3 LCPUFAs were significantly positively related to the ratio of subcutaneous to preperitoneal fat (subcutaneous/ preperitoneal) assessed by ultrasound at $6 \mathrm{wk}$ postpartum (Table 3).

By contrast, consistent negative associations were found among early breast milk AA and the total n-6 LCPUFAs and weight, ponderal index, BMI, and lean body mass (LBM, g) up to 4 mo postpartum, as well as among AA and total n-6 LCPUFAs and fat mass (g) up to $6 \mathrm{wk}$ postpartum, but not at 1 y of age (Table 4). Early breast milk n-6 LCPUFAs were significantly negatively related to individual SFT, e.g., triceps and suprailiac SFT $(r=-0.19, P=0.025 ; r=-0.17, P=0.047)$, the sum of four SFT measurements and percentage body fat at 6 wk postpartum $(r=-0.19, P=0.036 ; r=-0.18, P=0.041)$. The body compositional data analyses for n-6 LCPUFAs are presented in Supplementary Table S3 online.

The AA/DHA ratio in early breast milk was significantly negatively related to BMI and LBM $(\mathrm{g})$ at $6 \mathrm{wk}$ postpartum, but not at 4 mo or 1 y postpartum. The n-6/n-3 LCPUFA ratio in breast milk at $6 \mathrm{wk}$ postpartum was significantly negatively related to BMI and ponderal index at $6 \mathrm{wk}$ postpartum, and with waist circumference at 1 y postpartum (data not shown).

Neither the AA/DHA ratio nor the total n-6/total n-3 LCPUFAs ratio in early breast milk were significantly related to individual SFT or the sum of four SFT measurements from $6 \mathrm{wk}$ postpartum up to 1 y postpartum (data not shown).

\section{Late Breast Milk FA Profile (4 mo Postpartum) in Relation to Infant Growth and Body Composition}

DHA, EPA, and n-3 LCPUFA proportions in late breast milk collected at 4 mo postpartum were significantly negatively related to length at 1 y postpartum (e.g., EPA: adjusted $\beta$ $-12.43 \mathrm{~cm}$ (95\% confidence interval $-20.36,-4.231) ; r=-0.13$; $P=0.004, n=117)$, and for EPA and n-3 LCPUFA also with

Table 3. Final adjusted multiple-regression analysis and partial correlation coefficients on the effect of maternal breast milk $n-3$ fatty acid profile at early (6th wk postpartum) lactation on infant body composition

\begin{tabular}{|c|c|c|c|c|c|c|c|c|}
\hline & & & $\begin{array}{l}\text { Breast milk DHA 6th wk } \\
\text { postpartum }\end{array}$ & & $\begin{array}{l}\text { Breast milk EPA 6th wk } \\
\text { postpartum }\end{array}$ & & $\begin{array}{l}\text { Breast milkn-3 LCPUFAs } \\
\text { 6th wk postpartum }\end{array}$ & \\
\hline & & $n$ & Adjusted $\beta(95 \% \mathrm{Cl})$ & $r$ & Adjusted $\beta(95 \% \mathrm{Cl})$ & $r$ & Adjusted $\beta(95 \% \mathrm{Cl})$ & $r$ \\
\hline \multirow{3}{*}{$\begin{array}{l}\text { Sum of four SFT } \\
\text { measurements } \\
(\mathrm{mm})\end{array}$} & $6 w k$ & 152 & $0.08(-0.49,2.11)$ & 0.03 & $3.37(-2.39,9.13)$ & 0.08 & $0.50(-0.34,1.33)$ & 0.05 \\
\hline & $4 \mathrm{mo}$ & 148 & $1.55(0.12,2.98)$ & $0.12^{*}$ & $5.25(-1.15,11.66)$ & 0.11 & $0.90(-0.03,1.82)$ & 0.12 \\
\hline & $12 \mathrm{mo}$ & 141 & $1.43(0.01,2.84)$ & $0.16^{*}$ & $6.53(0.29,12.77)$ & $0.20^{*}$ & $0.91(0.00,1.82)$ & $0.18^{*}$ \\
\hline \multirow[t]{3}{*}{ Fat mass (g) } & $6 w k$ & 152 & $31.89(-44.31,108.09)$ & 0.06 & $119.76(-218.36,457.87)$ & 0.09 & $17.61(-31.36,66.58)$ & 0.07 \\
\hline & $4 \mathrm{mo}$ & 147 & $96.49(0.57,192.4)$ & 0.19 & $252.44(-174.72,679.60)$ & 0.16 & $50.34(-11.42,112.09)$ & 0.18 \\
\hline & $12 \mathrm{mo}$ & 141 & $125.02(-18.4,268.44)$ & 0.22 & $575.84(-56.7,1,208.39)$ & 0.23 & $78.88(-13.06,170.81)$ & 0.23 \\
\hline \multirow[t]{3}{*}{ Body fat (\%) } & $6 w k$ & 152 & $0.64(-0.37,1.66)$ & 0.02 & $2.72(-1.79,7.23)$ & 0.07 & $0.39(-0.26,1.05)$ & 0.04 \\
\hline & $4 \mathrm{mo}$ & 148 & $1.00(0.05,1.95)$ & $0.12^{*}$ & $3.29(-0.96,7.54)$ & 0.11 & $0.56(-0.05,1.18)$ & 0.12 \\
\hline & $12 \mathrm{mo}$ & 141 & $0.95(-0.01,1.9)$ & 0.16 & $4.16(-0.07,8.39)$ & 0.18 & $0.59(-0.03,1.20)$ & 0.17 \\
\hline \multirow[t]{3}{*}{ Ratio SC/PP fat } & $6 w k$ & 125 & $0.49(0.02,0.95)$ & $0.10^{*}$ & $1.66(-0.36,3.67)$ & 0.12 & $0.30(0.01,0.60)$ & $0.12^{*}$ \\
\hline & $4 \mathrm{mo}$ & 126 & $0.26(-0.27,0.78)$ & 0.09 & $0.58(-1.7,2.87)$ & 0.06 & $0.16(-0.17,0.49)$ & 0.09 \\
\hline & $12 \mathrm{mo}$ & 132 & $0.11(-0.14,0.36)$ & 0.04 & $0.66(-0.43,1.75)$ & 0.10 & $0.08(-0.08,0.24)$ & 0.06 \\
\hline
\end{tabular}

Data are presented as the regression coefficient of the fatty acid of interest ( $\beta$ ) along with the ( $95 \%$ confidence interval $(\mathrm{Cl}))$ and the partial correlation coefficient according to the Spearman's correlation coefficient $(r)$ of the fatty acid of interest. The asterisks $(*)$ indicate significant total model $P$ values of the (final) multivariable-adjusted analysis $(F$-test, analysis of covariance) and in the Spearman correlation coefficient $(* P<0.05)$.

DHA, docosahexaenoic acid; EPA, eicosapentaenoic acid; LCPUFA, long-chain polyunsaturated fatty acid; PP, preperitoneal; SC, subcutaneous; SFT, skinfold thickness. 


\section{Articles $\mid$ Much et al.}

Table 4. Final adjusted multiple-regression analysis and partial correlation coefficients on the effect of maternal breast milk $n-6$ fatty acid profile at early lactation (6th wk postpartum) on infant growth and growth indexes over the first year of life

\begin{tabular}{|c|c|c|c|c|c|c|}
\hline & & & Breast milk AA 6th wk postpartum & & $\begin{array}{l}\text { Breast milkn-6 LCPUFAs 6th wk } \\
\text { postpartum }\end{array}$ & \\
\hline & & $n$ & Adjusted $\beta(95 \% \mathrm{Cl})$ & $r$ & Adjusted $\beta(95 \% \mathrm{Cl})$ & $r$ \\
\hline \multirow[t]{3}{*}{ Weight (g) } & $6 w k$ & 152 & $-1,833.53(-2,926.5,-740.56)$ & $-0.32^{* *}$ & $-779.34(-1,201.38,-357.3)$ & $-0.36^{* * *}$ \\
\hline & $4 \mathrm{mo}$ & 148 & $-1,608.45(-2,978.68,-238.22)$ & $-0.23^{*}$ & $-687.65(-1,218.36,-156.95)$ & $-0.26^{*}$ \\
\hline & $12 \mathrm{mo}$ & 146 & $-1,410.28(-3,447.83,627.42)$ & -0.13 & $-261.16(-1,060.42,538.10)$ & -0.06 \\
\hline \multirow[t]{2}{*}{ Length (g) } & $6 w k$ & 152 & $-1.92(-6.19,2.35)$ & -0.11 & $-0.55(-2.21,1.12)$ & -0.12 \\
\hline & $12 \mathrm{mo}$ & 146 & $-0.97(-6.32,4.38)$ & -0.01 & $0.11(-1.98,2.20)$ & 0.03 \\
\hline \multirow[t]{3}{*}{ BMI $\left(\mathrm{kg} / \mathrm{m}^{2}\right)$} & $6 w k$ & 152 & $-4.88(-7.32,-2.44)$ & $-0.35^{* * *}$ & $-2.23(-3.16,-1.3)$ & $-0.41^{* * *}$ \\
\hline & $4 \mathrm{mo}$ & 148 & $-4.08(-6.81,-1.35)$ & $-0.25^{* *}$ & $-1.65(-2.71,-0.59)$ & $-0.27^{* *}$ \\
\hline & $12 \mathrm{mo}$ & 146 & $-1.93(-4.67,0.82)$ & -0.16 & $-0.52(-1.60,0.55)$ & -0.11 \\
\hline $\mathrm{PI}\left(\mathrm{kg} / \mathrm{m}^{3}\right)$ & 6 wk & 152 & $-7.60(-12.81,-2.40)$ & $-0.26^{* *}$ & $-3.70(-5.70,-1.71)$ & $-0.31^{* * *}$ \\
\hline \multirow{2}{*}{ LBM (g) } & $4 \mathrm{mo}$ & 147 & $-1,057.03(-2,004.02,-110.04)$ & $-0.23^{*}$ & $-491.69(-857.20,-126.18)$ & $-0.29^{* *}$ \\
\hline & $12 \mathrm{mo}$ & 141 & $-1,002.19(-2,394.59,390.20)$ & -0.12 & $-141.72(-686.50,403.05)$ & -0.05 \\
\hline
\end{tabular}

length at 4 mo postpartum (e.g., EPA: adjusted $\beta-7.84 \mathrm{~cm}$ (95\% confidence interval $-14.94,-0.73) ; r=-0.12 ; P=0.033$, $n=119)$. Breast milk proportions of DHA, EPA, and n-3 LCPUFAs at 4 mo postpartum were significantly positively related to ponderal index at 1 y postpartum, whereas breast milk DHA content was significantly positively associated with BMI at 1 y postpartum (e.g., breast milk DHA at 4 mo postpartum associated with BMI at 1 y postpartum: adjusted $\beta 0.86 \mathrm{~kg} /$ $\mathrm{m}^{2}$ (95\% confidence interval: $\left.0.11,1.62\right) ; r=-0.15 ; P=0.026$, $n=117)$. The AA/DHA and n-6/n-3 LCPUFAs ratios in breast milk taken at 4 mo postpartum were significantly negatively related to the abdominal preperitoneal fat layer as assessed by abdominal ultrasonography at 4 mo of age (data not shown). This relationship was not observed at $6 \mathrm{wk}$ or $1 \mathrm{y}$ postpartum.

No further significant or consistent relationships were observed between the FA profile in breast milk at 4 mo and infant clinical outcomes up to $1 \mathrm{y}$ of age, neither for the growth indexes nor for the measures of fat mass assessed by SFT measurements or ultrasound (data not shown).

\section{DISCUSSION}

The purpose of the present study was to investigate the independent relationships between n-6 LCPUFAs, n-3 LCPUFAs, and their ratios in human breast milk and infant body composition and growth during the first year of life. The association analyses yielded novel results on the differential effects of breast milk FAs from the n-3 FA family vs. the n-6 FA family on infant fat mass and indexes of growth. The central observation was that breast milk n-3 LCPUFAs at $6 \mathrm{wk}$ postpartum were positively associated with parameters of body fat in the offspring, arguing against the hypothesis that exposure to an increased supply of n-3 LCPUFA during early development may prevent an expansive adipose tissue growth.

The specific dietary intervention of the INFAT study was successful, as shown by higher n-3 LCPUFA proportions in breast milk from $6 \mathrm{wk}$ through 4 mo postpartum. Daily supplementation with $1,200 \mathrm{mg}$ LCPUFAs $(1,020 \mathrm{mg}$ DHA + $180 \mathrm{mg}$ EPA) and a concomitant moderate reduction in maternal AA intake led to a pronounced decrease in the breast milk n-6/n-3 LCPUFA ratio up to 4 mo postpartum, which was also clearly reflected by the FA profile in infant RBCs at the end of the dietary intervention.

An increased secretion of EPA and DHA into human breast milk upon supplementation with fish oil is in agreement with previous observations from n-3 LCPUFA supplementation RCTs during lactation (16-19). However, breast milk AA turned out to be nonresponsive to maternal diet, but tightly controlled, in the present study. This finding is consistent with previous reports from RCTs $(16,18-20)$, describing that n-3 LCPUFA supplementation during pregnancy and lactation does not decrease the proportion of AA in mature breast milk. Also, the concomitant moderate reduction in dietary AA intake during pregnancy in the present study (15) did not affect breast milk AA content. Indeed, breast milk AA is relatively uniform across populations on a worldwide basis $(\sim 0.24-1.0 \%$ of FAs) (21), suggesting a physiological and critical role of AA for infant growth and development.

The fact that we also did not observe any considerable correlation between maternal RBCs and breast milk AA levels gives further support to the suggestion that breast milk AA appears 
to be under strict physiological control. There is evidence that the slowly turning-over maternal body pools of AA are the major source of milk AA (22), although this association may be modified by the consumption of AA-rich foods/supplements by lactating mothers (in addition to extra n-3 LCPUFAs), which has been demonstrated to dose-dependently increase AA concentrations in breast milk lipids (23).

Breast milk EPA and DHA content constitute the highest variability of all FAs $(24,25)$, and breast milk concentrations are closely linked to maternal dietary EPA and DHA intake (26). However, little is currently known concerning the role of circulating LCPUFAs in breast milk for adipose tissue growth in the offspring, and data from RCTs are particularly scarce.

In a Danish trial, mothers with low habitual n-3 FA intake were randomized to receive either fish oil (supplying $0.62 \mathrm{~g}$ EPA and $0.79 \mathrm{~g}$ DHA per day) or olive oil from 0 to 4 mo of lactation, and the relationship between infant body composition, as measured by two-sided SFT investigations, and maternal RBC DHA as a biochemical measure of compliance was investigated (27). The maternal fish oil supplementation during lactation led to an increased BMI and waist circumference at $2.5 \mathrm{y}$ of age. Also, in a subgroup of 60 infants, BMI, waist circumference, triceps SFT, and percentage body fat at $2.5 \mathrm{y}$ of age were positively associated with the DHA content of maternal RBCs at the end of the intervention period in adjusted models. Of note, DHA content in a sample of breast milk taken at 4 mo postpartum was also significantly associated with BMI and waist circumference at $2.5 \mathrm{y}$, but no association with the sum of triceps + subscapular SFT was found (27). In the long-term follow-up of the children at $7 \mathrm{y}$ of age, however, none of the growth or body composition variables were correlated with maternal RBC DHA (28).

The Danish study is the only postnatal n-3 LCPUFA supplementation trial to date performing SFT measurements to determine percentage body fat and thus being able to discriminate between infant fat and LBM. However, the trial suffered from a large dropout rate in the $2.5 \mathrm{y}$ follow-up and exclusively addressed the issue in post hoc analyses.

In a trial from Norway investigating women randomized either to cod liver oil or corn oil from $18 \mathrm{wk}$ of pregnancy until 3 mo of lactation, Helland et al. found no correlation between the content of n-6 LCPUFAs (linolenic acid, AA) in breast milk and BMI at the age of $7 \mathrm{y}$. By contrast, they observed that the content of $\alpha$-linolenic acid in breast milk collected at $4 \mathrm{wk}$ and 3 mo after birth was positively correlated with later BMI (29).

In our study, we found significant positive associations between early, but not late, breast milk LCPUFAs from the three series of different measures of infant fat mass across all time points.

This suggests that, contrary to our original hypothesis, an increased supply of n-3 FAs during critical periods of adipose tissue development in the newborn via breast milk stimulates fat accumulation in early postnatal life. This unexpected finding supports our previous null findings in adipose tissue growth between the intervention and control groups (15).

This finding is also in contrast with former animal data linking an increased n-3 LCPUFA supply with reduced fat cell differentiation and accumulation in adult rodents and in vitro studies $(8,9)$, and in offspring whose mothers were fed a diet high in n-3 FAs during the pregnancy/lactation period (8). However, in almost all cases, studies in animals have failed to restrict the intervention to the perinatal period, making it impossible to examine the effects of n-3 FA exposure during this critical period.

Another interesting finding of the present study was that early breast milk n-6 LCPUFAs, including AA, showed consistent negative associations with infant body weight, growth parameters such as BMI and ponderal index, and LBM (g) up to 4 mo postpartum, but not at $1 \mathrm{y}$ of age. Early breast milk n-6 LCPUFAs were also significantly negatively related to individual SFT (e.g., triceps, suprailiac SFT), the sum of the four SFT measurements and percentage body fat up to $6 \mathrm{wk}$ postpartum.

This suggests that early breast milk FAs from the n-6 FA family serve as important regulating factors for growth in early postnatal life, and particularly of fat growth and LBM. Due to the low variability of AA contents in breast milk, the ingested volume as defined by the daily amount of milk intake by the breastfed infant might play a critical role in growth-regulatory effects of AA. Further studies are needed to look into such quantitative aspects.

By contrast, early breast milk FAs from the n-3 FA family, thus DHA, EPA, or total n-3 LCPUFAs, were not associated with growth indexes or LBM up to the first year of life, suggesting different functional roles of LCPUFAs from the $n-6$ vs. $n-3$ FA series in postnatal growth. Of note, the AA/DHA and the total n-6/n-3 LCPUFAs ratios were significantly negatively associated with BMI at $6 \mathrm{wk}$ postpartum, but were not associated with any of the other outcomes under study up to 1 y postpartum.

Our latter finding is supported by a very recent analysis of the Copenhagen Prospective Study on Asthma and Childhood, a cohort study primarily designed to assess children born to atopic mothers (30). Pedersen et al. observed no associations between breast milk n-6/n-3 PUFA ratio at $\sim 3$ wk postpartum and body composition assessed by dual energy X-ray absorptiometry between the age of 2-7 y. Of note, the Danish study found an inverse relationship between breast milk DHA and parameters of child growth, observed by changes in the timing of adiposity rebound, BMI from 2 to $7 \mathrm{y}$, and body fat mass, suggesting that high DHA supply to the breastfed infant might delay the age at adiposity rebound. These results have to be confirmed in longterm interventional trials in a nonatopic population.

A strength of the INFAT study is that the breast milk samples were collected at two different time points over the breastfeeding period, which may be a reliable reflection of infant's LCPUFAs supply throughout lactation. This enabled us to investigate the association of both the early and the late breast milk LCPUFA profiles with infant developmental outcome at different postnatal ages. Thereby, we unraveled marked differences in the regulatory function of breast milk LCPUFAs, with early breast milk n-3 LCPUFAs being the more important driver of postnatal fat mass growth.

It is rather unlikely that LCPUFAs from a source other than breast milk have distorted the associations between the FA 
composition of the breast milk and infant fat mass. Because $95 \%$ of the women in both groups in the present study decided to breastfeed their infants and the duration of exclusive breastfeeding was on average $20 \mathrm{wk}$, the proportion of FAs present in breast milk is expected to reflect the amount of LCPUFAs ingested by the infants.

We acknowledge that the data presented are associations, which may not indicate cause-and-effect relationships. Moreover, the reported associations between breast milk n-3 LCPUFAs and fat mass were significant, but the correlation coefficients were found to be low $(3-20 \%$ of the variance in SFT), suggesting that other factors are more likely to contribute to adipose tissue growth in early infancy.

We cannot exclude that genetic variants have modified the association between fish oil intake and DHA proportions in human milk. Recently, a Dutch study provided evidence that a high fish (or fish oil) intake in women homozygous for the minor allele for a certain FADS1/2 gene variant was able to compensate for their lower DHA proportions in plasma phospholipids but not in their milk, as compared with carriers of the major allele (31). However, such gene-diet interactions in LCPUFA metabolism remain to be confirmed in larger studies.

\section{Conclusion}

The results of our study suggest that breast milk n-3 LCPUFAs at $6 \mathrm{wk}$ postpartum appear to stimulate fat mass growth over the first year of life, whereas AA seems to be involved in the regulation of overall growth, especially in the early postpartum period. The LCPUFA ratio in breast milk does not seem to be a critical determinant in infant adiposity development. However, a longer follow-up of growth parameters is required to assess potential long-term effects of LCPUFAs in breast milk on these clinical outcomes.

\section{METHODS}

\section{Study Design, Subjects, and Dietary Intervention}

Data were collected within the INFAT study, an open-label randomized controlled dietary intervention trial with two parallel arms. The INFAT trial was designed as a proof-of-concept study to test the hypothesis that lowering the $n-6 / n-3$ long-chain FA ratio in the diet of pregnant/breastfeeding women may reduce the expansion of adipose tissue growth early in life and may, thereby, represent a completely novel approach for the primary prevention of childhood obesity. The study rationale and design, inclusion and exclusion criteria, and the clinical results regarding infant fat mass up to $1 \mathrm{y}$ of age have been published elsewhere $(13,15)$.

In brief, 208 healthy women around their 14th wk of gestation were enrolled into the INFAT study and randomly assigned either to a dietary intervention (1,200 mg n-3 LCPUFAs per day (1,020 mg DHA and $180 \mathrm{mg}$ EPA) + instructions to normalize their AA intake to $90 \mathrm{mg} / \mathrm{d}$ ) or a control group (healthy diet according to the current guidelines in Germany) from the 15th wk of gestation until 4 mo postpartum (13).

The primary outcome was infant fat mass estimated by individual and summed SFT measurements up to 12 mo postpartum. Analyses of the primary outcome did not verify the original hypothesis because we found no thinner SFT (i.e., lower fat mass) in the infants whose mothers received the dietary intervention as compared with infants of the control group.

In this report, a preplanned secondary analysis of the initial study protocol was performed. The objective of this analysis was to determine the effects of circulating LCPUFAs in infant blood and in breast milk samples as potential determinants of infant body and adipose tissue growth (13). Written informed consent was obtained from all the subjects before starting the study. The study protocol was approved by the ethical committee of the Technical University of Munich (no. $1479 / 06 / 2006 / 2 / 21)$.

\section{Data, Blood and Milk Sample Collection, and Measurements}

Maternal breast milk samples were collected in the morning after an overnight fast in a standardized manner in the study center by breast pump at $6 \mathrm{wk}$ and 4 mo postpartum, aliquoted and immediately stored at $-86^{\circ} \mathrm{C}$ until analysis.

Infant blood was collected by a pediatrician by venipuncture at 4 and 12 mo postpartum in a subsample in EDTA-containing tubes. Samples were immediately centrifuged to separate erythrocytes and plasma. The erythrocytes were washed with $0.9 \% \mathrm{NaCl}$ solution, aliquoted, and stored at $-86^{\circ} \mathrm{C}$ until analysis.

Weight, length, and head circumference of the infant were measured at $6 \mathrm{wk}$ and 4 and 12 mo postpartum (15). Infant weight-forlength $(\mathrm{kg} / \mathrm{m})$, BMI $\left(\mathrm{kg} / \mathrm{m}^{2}\right)$, and ponderal index $\left(\mathrm{kg} / \mathrm{m}^{3}\right)$ were calculated. Longitudinal SFT measurements were performed at 3-5 d postpartum, $6 \mathrm{wk}$ postpartum, and 4 and 12 mo postpartum at four body sites with a caliper in a standardized manner as reported previously (15). Body fat (\%) was calculated according to the method of Weststrate et al. (32).

Abdominal subcutaneous fat and preperitoneal fat thickness were measured at $6 \mathrm{wk}$ and 4 and 12 mo postpartum by abdominal ultrasonography (15). The technique used was a modification of the method recently described by Holzhauer and colleagues (33).

FA Analysis in Breast Milk and RBCs

The analysis of FAs was performed at the Laboratory of Lipid Research, Danone Research, Centre for Specialised Nutrition, Friedrichsdorf, Germany, according to established protocols $(34,35)$. FA values are presented as percentage of FA/total FA. All analyses were done in duplicate.

\section{Statistical Analysis}

Changes in breast milk FA profile over time were determined by using the Wilcoxon test. Differences in FA values between the groups were compared using the Mann-Whitney $U$-test. Bonferroni correction of $P$ values was applied to correct for multiple comparisons.

To determine correlations between the FA profile in breast milk and infant blood, Spearman's correlation coefficients were used. To clarify potential influences of breast milk FA profile at different stages of lactation on infant body composition within the first year of life, multipleregression models (analysis of covariance, $F$-test) and partial correlations (Spearman's $r$ ) were performed for the early ( $6 \mathrm{wk}$ postpartum) and late (4 mo postpartum) breast milk FA profile. Results were corrected for gestational age, parity, infant sex, group, ponderal index at birth, and breastfeeding status in the analysis of the infants at $6 \mathrm{wk}, 4$ mo, and $1 \mathrm{y}$. The FA profiles in these body compositional analyses comprise DHA, EPA, n-3 LCPUFA, AA, and n-6 LCPUFA content in breast milk, as well as AA/DHA and n-6/n-3 LCPUFA ratios. Data are shown for selected FAs only. Statistical analyses were performed using software package R version 2.8.1 (R Foundation for Statistical Computing, Vienna, Austria) and PASW software (version 17.0; SPSS, Chicago, IL). A two-sided $P$ value $<0.05$ was considered statistically significant.

\section{SUPPLEMENTARY MATERIAL}

Supplementary material is linked to the online version of the paper at http:// www.nature.com/pr

\section{ACKNOWLEDGMENTS}

The authors thank Angelika Müller-Green and Michael Möbius from Danone Research for their technical assistance and performance of the fatty acid analysis.

\section{STATEMENT OF FINANCIAL SUPPORT}

D.M., S.B., C.V., D.S., E.-M.S., E.H., B.L.B., and U.A.-G. declare financial support to their institutions for the submitted work from Else Kröner-Fresenius 
Foundation, Bad Homburg; the International Unilever Foundation, Hamburg; the European Union-funded EARNEST (Early Nutrition Programming Project) consortium (FOOD-CT-2005-007036), administratively facilitated by Frank Wiens; the German Ministry of Education and Research via the Competence Network on Obesity (Kompetenznetz Adipositas, GF-GFGI01120708); and Danone Research, Centre for Specialised Nutrition, Friedrichsdorf, Germany. Trial registry: ClinicalTrial.gov, number ID NCT00362089, http://www. clinicaltrials.gov/ct2/show/NCT00362089.

Disclosure: The analysis of fatty acids was performed by the laboratory of Lipid Research, Danone Research, Centre for Specialised Nutrition using coded samples. N.B. and G.B. are employees at Danone Research. There was no intervention from any sponsor with any of the research aspects of the study, including study design, intervention, data collection, data analysis, and interpretation, as well as writing of the manuscript. The other authors declared no conflict of interest.

\section{REFERENCES}

1. Lauritzen L, Carlson SE. Maternal fatty acid status during pregnancy and lactation and relation to newborn and infant status. Matern Child Nutr 2011;7:Suppl 2:41-58.

2. Kovács A, Funke S, Marosvölgyi T, Burus I, Decsi T. Fatty acids in early human milk after preterm and full-term delivery. J Pediatr Gastroenterol Nutr 2005;41:454-9.

3. Szabó E, Boehm G, Beermann C, et al. Fatty acid profile comparisons in human milk sampled from the same mothers at the sixth week and the sixth month of lactation. J Pediatr Gastroenterol Nutr 2010;50:316-20.

4. Xie L, Innis SM. Genetic variants of the FADS1 FADS2 gene cluster are associated with altered (n-6) and (n-3) essential fatty acids in plasma and erythrocyte phospholipids in women during pregnancy and in breast milk during lactation. J Nutr 2008;138:2222-8.

5. Tanaka T, Shen J, Abecasis GR, et al. Genome-wide association study of plasma polyunsaturated fatty acids in the InCHIANTI Study. PLoS Genet 2009;5:e1000338.

6. Delgado-Noguera M, Calvache J, Bonfill C. Supplementation with long chain polyunsaturated fatty acids (LCPUFA) to breastfeeding mothers for improving child growth and development. Cochrane Database Syst Rev 2010:CD007901.

7. Ailhaud G, Guesnet P. Fatty acid composition of fats is an early determinant of childhood obesity: a short review and an opinion. Obes Rev 2004;5:21-6.

8. Massiera F, Saint-Marc P, Seydoux J, et al. Arachidonic acid and prostacyclin signaling promote adipose tissue development: a human health concern? J Lipid Res 2003;44:271-9.

9. Oosting A, Kegler D, Boehm G, Jansen HT, van de Heijning BJ, van der Beek EM. N-3 long-chain polyunsaturated fatty acids prevent excessive fat deposition in adulthood in a mouse model of postnatal nutritional programming. Pediatr Res 2010;68:494-9.

10. Muhlhausler BS, Gibson RA, Makrides M. The effect of maternal omega-3 long-chain polyunsaturated fatty acid ( $\mathrm{n}-3$ LCPUFA) supplementation during pregnancy and/or lactation on body fat mass in the offspring: a systematic review of animal studies. Prostaglandins Leukot Essent Fatty Acids 2011;85:83-8.

11. Muhlhausler BS, Miljkovic D, Fong L, Xian CJ, Duthoit E, Gibson RA. Maternal omega-3 supplementation increases fat mass in male and female rat offspring. Front Genet 2011;2:48.

12. Muhlhausler BS, Gibson RA, Makrides M. Effect of long-chain polyunsaturated fatty acid supplementation during pregnancy or lactation on infant and child body composition: a systematic review. Am J Clin Nutr 2010;92:857-63.

13. Hauner H, Vollhardt C, Schneider KT, Zimmermann A, Schuster T, Amann-Gassner $\mathrm{U}$. The impact of nutritional fatty acids during pregnancy and lactation on early human adipose tissue development. Rationale and design of the INFAT study. Ann Nutr Metab 2009;54:97-103.

14. Ailhaud G, Massiera F, Weill P, Legrand P, Alessandri JM, Guesnet P. Temporal changes in dietary fats: role of $n-6$ polyunsaturated fatty acids in excessive adipose tissue development and relationship to obesity. Prog Lipid Res 2006;45:203-36.
15. Hauner H, Much D, Vollhardt C, et al. Effect of reducing the n-6:n-3 longchain PUFA ratio during pregnancy and lactation on infant adipose tissue growth within the first year of life: an open-label randomized controlled trial. Am J Clin Nutr 2012;95:383-94.

16. Fidler N, Sauerwald T, Pohl A, Demmelmair H, Koletzko B. Docosahexaenoic acid transfer into human milk after dietary supplementation: a randomized clinical trial. J Lipid Res 2000;41:1376-83.

17. Helland IB, Saugstad OD, Smith L, et al. Similar effects on infants of $n-3$ and $n-6$ fatty acids supplementation to pregnant and lactating women. Pediatrics 2001;108:E82.

18. Jensen CL, Voigt RG, Prager TC, et al. Effects of maternal docosahexaenoic acid intake on visual function and neurodevelopment in breastfed term infants. Am J Clin Nutr 2005;82:125-32.

19. Lauritzen L, Jørgensen MH, Mikkelsen TB, et al. Maternal fish oil supplementation in lactation: effect on visual acuity and n-3 fatty acid content of infant erythrocytes. Lipids 2004;39:195-206.

20. Dunstan JA, Mitoulas LR, Dixon G, et al. The effects of fish oil supplementation in pregnancy on breast milk fatty acid composition over the course of lactation: a randomized controlled trial. Pediatr Res 2007;62: 689-94.

21. Brenna JT, Varamini B, Jensen RG, Diersen-Schade DA, Boettcher JA, Arterburn LM. Docosahexaenoic and arachidonic acid concentrations in human breast milk worldwide. Am J Clin Nutr 2007;85:1457-64.

22. Del Prado M, Villalpando S, Elizondo A, Rodríguez M, Demmelmair H, Koletzko B. Contribution of dietary and newly formed arachidonic acid to human milk lipids in women eating a low-fat diet. Am J Clin Nutr 2001;74:242-7.

23. Weseler AR, Dirix CE, Bruins MJ, Hornstra G. Dietary arachidonic acid dose-dependently increases the arachidonic acid concentration in human milk. J Nutr 2008;138:2190-7.

24. Smit EN, Martini IA, Mulder H, Boersma ER, Muskiet FA. Estimated biological variation of the mature human milk fatty acid composition. Prostaglandins Leukot Essent Fatty Acids 2002;66:549-55.

25. Yuhas R, Pramuk K, Lien EL. Human milk fatty acid composition from nine countries varies most in DHA. Lipids 2006;41:851-8.

26. Makrides M, Neumann MA, Gibson RA. Effect of maternal docosahexaenoic acid (DHA) supplementation on breast milk composition. Eur J Clin Nutr 1996;50:352-7.

27. Lauritzen L, Hoppe C, Straarup EM, Michaelsen KF. Maternal fish oil supplementation in lactation and growth during the first 2.5 years of life. Pediatr Res 2005;58:235-42.

28. Asserhøj M, Nehammer S, Matthiessen J, Michaelsen KF, Lauritzen L. Maternal fish oil supplementation during lactation may adversely affect long-term blood pressure, energy intake, and physical activity of 7-yearold boys. J Nutr 2009;139:298-304.

29. Helland IB, Smith L, Blomén B, Saarem K, Saugstad OD, Drevon CA. Effect of supplementing pregnant and lactating mothers with $n-3$ verylong-chain fatty acids on children's IQ and body mass index at 7 years of age. Pediatrics 2008;122:e472-9.

30. Pedersen L, Lauritzen L, Brasholt M, Buhl T, Bisgaard H. Polyunsaturated fatty acid content of mother's milk is associated with childhood body composition. Pediatr Res 2012;72:631-6.

31. Moltó-Puigmartí C, Plat J, Mensink RP, et al. FADS1 FADS2 gene variants modify the association between fish intake and the docosahexaenoic acid proportions in human milk. Am J Clin Nutr 2010;91:1368-76.

32. Weststrate JA, Deurenberg P. Body composition in children: proposal for a method for calculating body fat percentage from total body density or skinfold-thickness measurements. Am J Clin Nutr 1989;50:1104-15.

33. Holzhauer S, Zwijsen RM, Jaddoe VW, et al. Sonographic assessment of abdominal fat distribution in infancy. Eur J Epidemiol 2009;24:521-9.

34. Lepage G, Roy CC. Improved recovery of fatty acid through direct transesterification without prior extraction or purification. J Lipid Res 1984;25:1391-6

35. Beermann C, Möbius M, Winterling N, Schmitt JJ, Boehm G. sn-position determination of phospholipid-linked fatty acids derived from erythrocytes by liquid chromatography electrospray ionization ion-trap mass spectrometry. Lipids 2005;40:211-8. 